\title{
Biopsy of Musculoskeletal Tumors; Current Concepts Review
}

\author{
Khodamorad Jamshidi ${ }^{1, "} ;$ Abolfazl Bagherifard ${ }^{1}$ \\ ${ }^{1}$ Bone and Joint Reconstruction Research Center, Shafa Orthopedic Hospital, Iran University of Medical Sciences, Tehran, IR Iran \\ ${ }^{*}$ Corresponding author: Khodamorad Jamshidi, Bone and Joint Reconstruction Research Center, Shafa Orthopedic Hospital, Iran University of Medical Sciences, Tehran, IR Iran. Tel: \\ +98-2133542010, Fax:+98-2133542020, E-mail: jamshidi_k@yahoo.com
}

Received: June 22, 2014; Revised: September 15, 2014; Accepted: December 20, 2014

\begin{abstract}
Context: Appropriate management of soft-tissue and bone tumor needs correct diagnosis. Sometimes, diagnosis can be made by history, physical examination and imaging. An incompetently performed biopsy not only may fail in making the proper diagnosis, but also has a negative impact on survival and local control.

Evidence Acquisition: To reach the most accurate information in the field of biopsy of the musculoskeletal tumor, based on the intensive literature review of the most prestigious journals and textbooks in the field of the musculoskeletal tumor surgery and our experiences in the only referral center in our country for more than 25 years, this current concept review was prepared.

Results: Fine needle aspiration (FNA) has the highest accuracy in homogenous tumor as well as malignant myeloma and metastatic carcinoma. Core needle biopsy (CNB) is usually selected as the first biopsy modality. Incisional biopsy is indicated in difficult cases when a larger specimen is necessary for diagnosis. In malignant bone tumors, soft tissue component is as much representative as bony component.

Conclusions: Biopsy should be considered as a final diagnostic procedure. Not all bone and soft tissue lesions need biopsy. FNA, CNB and incisional biopsy should be performed as their precisely defined indications.
\end{abstract}

Keywords: Bone; Soft Tissue; Tumor; Biopsy

\section{Context}

Biopsy is the concluding stage in the diagnosis of bone and soft-tissue tumors and should be delayed until clinical evaluation and completion of imaging studies. In 1958, Jaffe stated that biopsy should be considered as the final diagnostic procedure, not as a shortcut to diagnosis (1). In typical benign lesions, biopsy is not needed. Yet biopsy is indicated in malignant and uncertain lesions to confirm or classify the diagnosis before treatment. Biopsy is usually a simple procedure. However, it is necessary to determine which part of the lesion is representative and the appropriate surgical approach must be carefully thought through. An incompetently performed biopsy not only may fail in making the proper diagnosis, but also has a negative impact on survival and local control (2). In 1996, Mankin et al. (3) performed a study on 597 patients who had biopsies done for bone and soft tissue sarcomas. They found an error rate of $13.5 \%$ in the diagnoses made by biopsies and a complication rate of $15.9 \%$ with $3 \%$ unnecessary amputations. These events occurred more frequently when the biopsies were performed in the referring institution rather than an oncology center. However, there is no evidence that a properly performed biopsy causes acceleration of tumor growth, metastatic dissemination or increasing rate of local recurrence. However, it is rational to suppose that biopsy increases the rate of local recurrence, which could be related to the width of biopsy tract and adequacy of hemostasis (4). Biopsy tissue can be obtained through a core-needle biopsy, an incisional biopsy or an excisional biopsy and biopsy cells can be obtained through fine needle aspiration (4). The purpose of this paper was to review the current concepts in biopsy of musculoskeletal tumors.

\section{Evidence Acquisition}

During 25 years, more than 15000 surgeries were done for patients with musculoskeletal tumors in the department of tumor surgery of Shafa orthopedic hospital, which is the only referral center in our country. Based on these extensive experiences we performed a comprehensive literature review of the related published papers in the most prestigious journals and tumor surgery textbooks to define the most accurate methods, indications and complications of biopsy procedure in the field of musculoskeletal tumor surgery.

\section{Results}

\subsection{Natural History of Musculoskeletal Tumors}

Musculoskeletal tumors usually arise from mesenchymal elements and have a unique characteristic. These lesions grow in a centripetal fashion and the most immature part is found at the growing edge. With growth of

Copyright (C) 2015, Iran University of Medical Sciences. This is an open-access article distributed under the terms of the Creative Commons Attribution-NonCommercial 4.0 International License (http://creativecommons.org/licenses/by-nc/4.0/) which permits copy and redistribute the material just in noncommercial usages, provided the original work is properly cited. 
tumor, normal connective tissue surrounding the tumor is compressed to form the capsule. The reactive zone is formed between capsule and normal surrounding tissue. It is composed of induced proliferation of mesenchymal tissue, neovasculature and inflammatory cells. The differentiation of mesenchymal tissue depends on the location of lesion. Soft tissue lesions simulate a fibrous reaction and intraosseous lesions simulate bone-forming reaction. Unlike sarcomas, carcinomas infiltrate directly into surrounding tissue and do not form the capsule or reactive zone (2-6).

\subsection{Position of the Biopsy Site}

Bone and soft tissue tumors are heterogeneous, so multiple samples are required to establish a diagnosis. Carcinomas, in contrast, are homogenous and a single tissue core or aspirate is sufficient for diagnosis. Before biopsy, imaging studies should be evaluated to address the following questions:

1) Which part or parts of the lesion are representative?

2) What is the safest anatomic route to the lesion? This is particularly important because the biopsy tract is contaminated and must be resected during the operation, so it should not violate more than one anatomic compartment and must be away from the vital structures such as neurovascular bundles. It is also crucial for the biopsy to be performed in the line of definite surgery (1).

\subsection{Biopsy Techniques}

\subsubsection{Fine Needle Aspiration Biopsy (FNAB) or Fine Nee- dle Aspiration Cytology (FNAC)}

FNA does not include incision. A fine, hollow needle is inserted into the mass directly for obtaining the sample. This procedure is mostly used for cytology examination of the aspirate (FNAC). There could be the possibility of histological tissue evaluation (FNAB) (7). Initially, FNA of mesenchymal tumors was criticized, because the amount of biopsy material was not enough to evaluate tissue architecture and for ancillary studies such as cytogenetic, molecular or immunohistochemical investigations. FNA has been reported to be a reliable technique for diagnosis of soft tissue sarcoma and provides sufficient tissue for ancillary studies $(8,9)$. This procedure has the highest accuracy in homogenous tumors as in the case of multiple myeloma and metastatic carcinoma. It can be used for local or distant recurrence where the cytology findings can be compared with prior histology specimens (10).

\subsubsection{Core Needle Biopsy (CNB)}

A14-gauge needle is inserted via a small puncture wound into the palpable mass directly or with the guidance of CT (Computed Tomography) or ultrasound. The site of insertion of the needle should be in the line of definite surgical incision (Figure 1). Multiple cores in different di- rections should be obtained. It provides a core of tissue with a $2 \mathrm{~mm}$ diameter and a maximum length of $20 \mathrm{~mm}$ (Figure 2). The architecture of tissue is well preserved for histological diagnosis and ancillary analysis. CNB is usually accomplished as the first biopsy modality. Open biopsy is performed when the sample is not representative. It is useful for deep-seated lesions like pelvis and spine and performed under CT guidance (11). Less morbidity and fewer complication rates have been reported for percutaneous biopsy ranging between $0 \%$ and $17 \%$, most commonly hematoma, bleeding and infection $(2,5,10)$.

Figure 1. Photograph of the Planning for Core Needle Biopsy From the Distal Femur With Medial Bump.

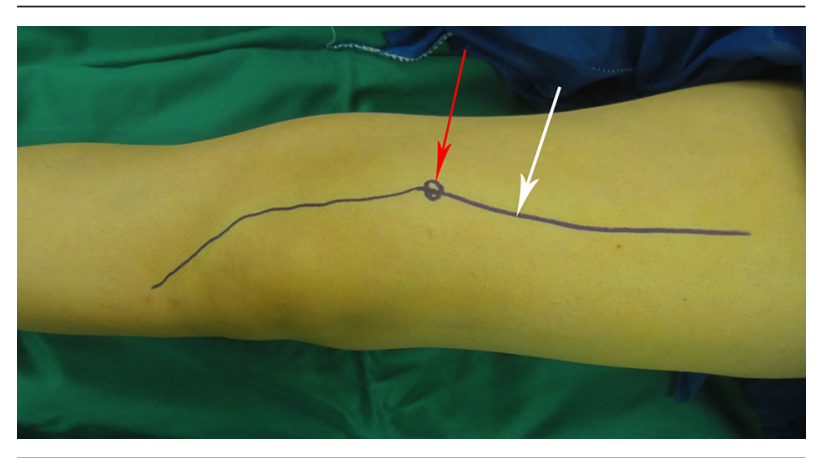

The definite surgical incision (white arrow) is designed for excision of biopsy tract (red arrow).

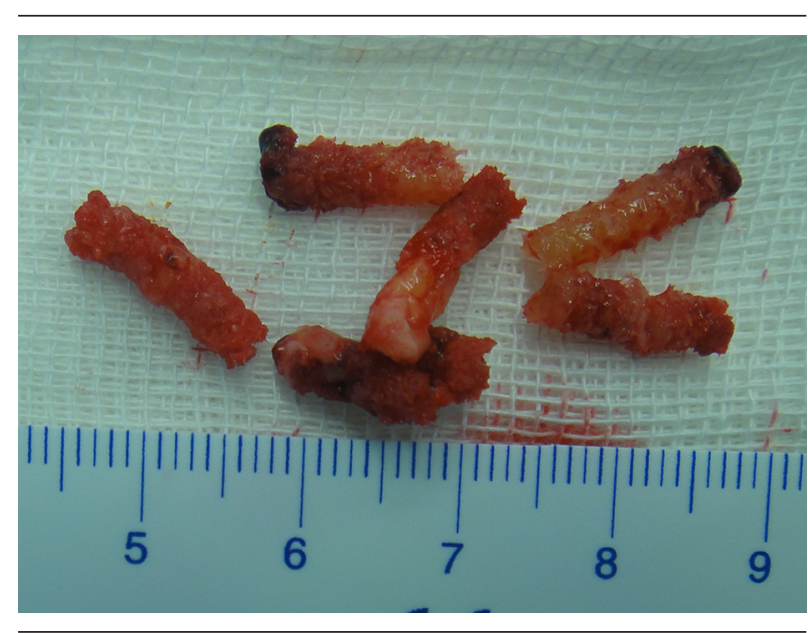

Figure 2. Photograph of Multiple Core of Tissue Obtained From Different Directions

\subsubsection{Incisional Biopsy}

In difficult cases when imaging studies are not conclusive or the sample obtained from CNB is inadequate and a large specimen is necessary for diagnosis, incisional biopsies are indicated (11). Imaging helps to determine the local extension of the lesion and presence of metastatic disease. Biopsy, especially incisional biopsy may change the imaging feature of the lesion and therefore alter 
the interpretation of imaging studies and has to be performed as the last procedure of diagnosis. Furthermore, staging studies determine the appropriate approach to the tumor and indicate which part of the tumor is representative (Figure 3 ). It should be performed with frozen section to ensure that appropriate tissue has been obtained. If a benign diagnosis is confirmed, definite surgery can be completed in the same stage. It is best to use the smallest longitudinal incision compatible with obtaining adequate sample. The samples should be taken from the periphery of the tumors because of frequent central necrosis (12). Hematoma formation in biopsy wound increases the risk of local spread of the tumor cells and logically increases the rate of local recurrence. A tourniquet should not be used routinely for this procedure, because the bleeding vessel is hardly seen and hemostasis is difficult to achieve. If a tourniquet is used to exsanguinate the limb, wrapping with Esmarch bandage is contraindicated, because this may push the tumor cell into the proximal part of the limb and even to the blood stream. Before wound closure, the tourniquet should be deflated to accomplish precise hemostasis. If the drain path is contaminated, it has to be excised. The entry of drain should be positioned in the line of incision and close to it i.e. no more than $1 \mathrm{~cm}$.

Figure 3. T2-Weighted fat Suppression MRI of the Distal Femur of a 16-Year-Old Patient.

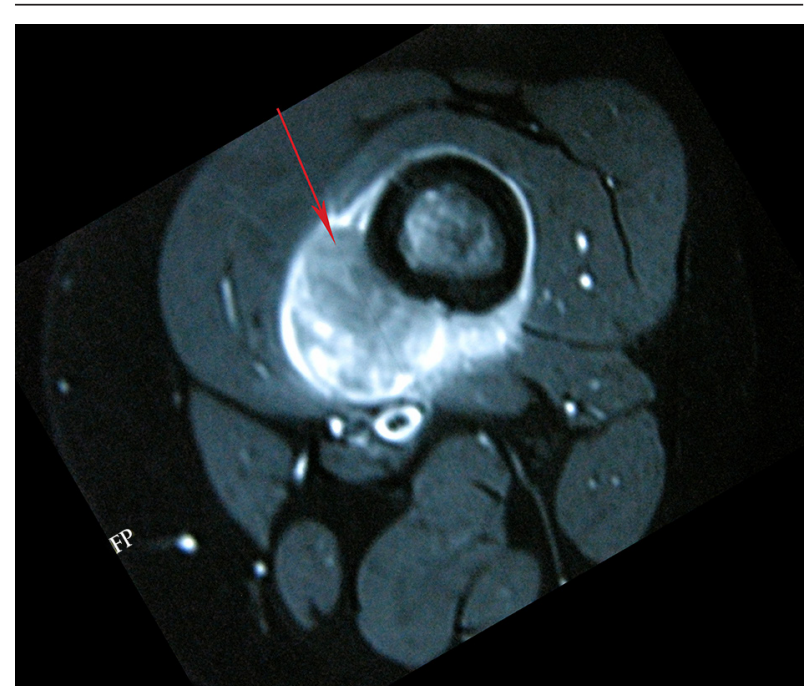

Old boy with osteosarcoma showing isosignal intensity relative to muscle of the tumor (red arrow) is appropriate for biopsy.

\subsubsection{Bone biopsy}

Many typical benign bone lesions do not need biopsy, such as osteoid osteoma, simple bone cyst, nonossifying fibroma, osteochondroma, chondroma and fibrous dysplasia. In malignant bone tumors, the soft tissue component is as much representative as the bony component (8). The specimen should be obtained by knife or large head curette to avoid crushing the tissue's texture. Disrupting the cortex increases the risk of pathologic fracture and only when the lesion is purely intraosseous, a cortical window has to be made and the shape should be considered carefully to minimize the risk of pathologic fracture. Clark et al. reported that oblong windows with rounded ends provided the lowest risk of pathologic fracture. They also emphasized that increasing the width of window decreased the strength of bone, while increasing the length did not (12).

The most common location of primary bone sarcoma is distal femur. Depending on the extraosseous extension of the lesion, antromedial or antrolateral longitudinal incision is selected (Figure 4). Neurovascular bundle, rectus femoris and knee joint should not be violated. After a skin incision directly through vastus medialis or vastus lateralis muscle the sample is obtained. The second most common site of bone sarcoma is proximal tibia. Anteromedial or anterolateral longitudinal incision is performed and corresponds to the soft-tissue component of the tumor (Figure 5). In upper limbs, the proximal humerus is the most common site of bone sarcomas. Usually the deltopectoral approach is selected for wide resection of the proximal humerus tumors. A transdeltoid approach through the anterior third of the muscle is used to avoid violating neurovascular bundle, the cephalic vein and the pectoralis major, for soft-tissue reconstruction (Figure 6).

Figure 4. Photograph of the Planning for Incisional Biopsy From the Distal Femur With Lateral Extraosseous Component

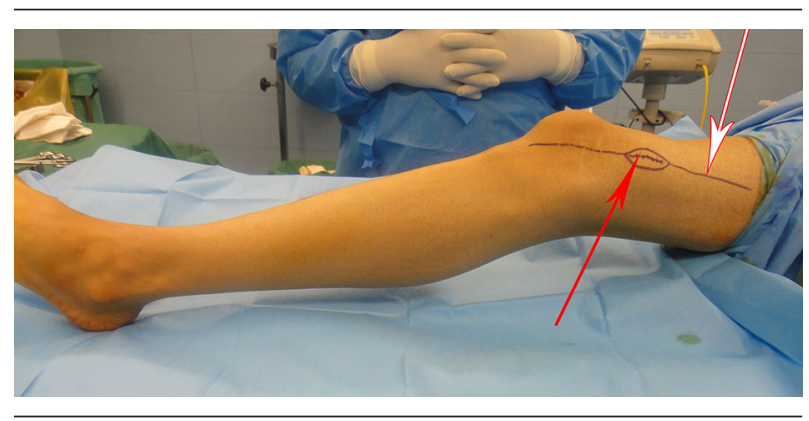

The definite surgical incision (white arrow) is designed for excision of biopsy incision (red arrow) and tract.

Figure 5. Photograph of the Planning for the Incisional Biopsy From the Proximal Tibia With Medial Extraosseous Component

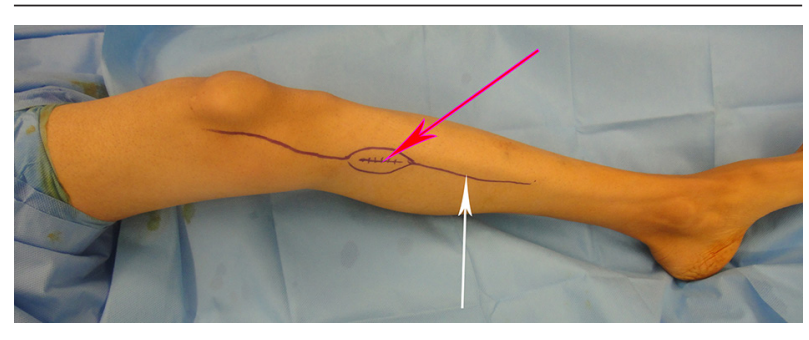

The definite surgical incision (white arrow) is designed for excision of biopsy incision (red arrow) and tract. 
Figure 6. Photograph of the Planning for Incisional Biopsy From the Proximal Humerus

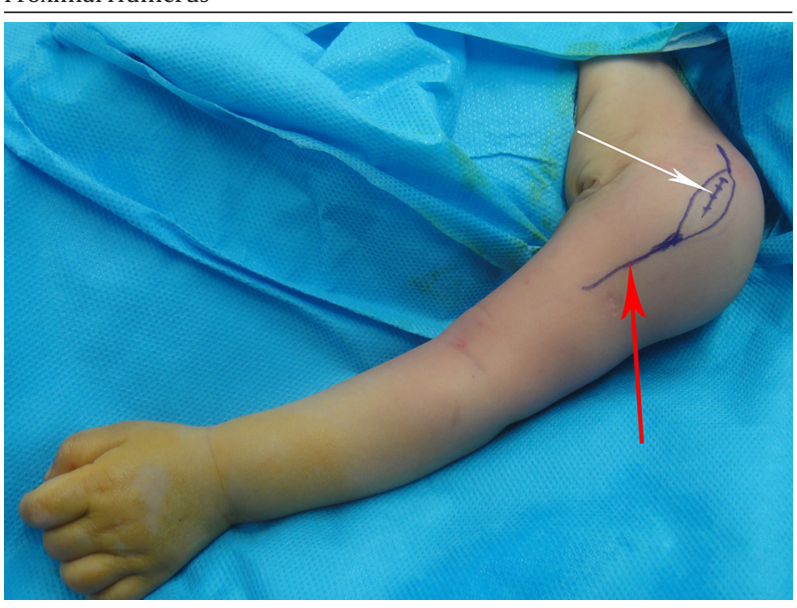

The definite surgical incision (red arrow) is designed for excision of the biopsy incision (white arrow) and tract.

\subsubsection{Soft Tissue Biopsy}

Benign soft tissue tumors are more common than soft tissue sarcomas with a ratio of at least 100:1(12). Many common benign soft tissue tumors such as lipoma and hemangioma do not need to be biopsied or removed. Lipoma, hemangioma, ganglion and popliteal cyst, PVNS and fibromatosis can be differentiated by MRI and do not need biopsy. In general, if a soft tissue mass largest diameter is $<5$ $\mathrm{cm}$ and or if it is superficial and does not involve the fascia, it can be excised by excisional biopsy (13). Although, biopsy is the only diagnostic procedure for some soft tissue masses, it is only indicated when clinical and imaging studies are not conclusive or when soft-tissue must be removed. The most common pitfall is the excision of mass before adequate imaging was performed because of the cost of imaging studies and the rational that the mass should be excised any way. Before biopsy, imaging studies should be completed. The biopsy site should be planned directly over the lump at the point, where the tumor is closest the skin and in the line of surgical incision of the definite surgery. Kasraian S, et al. compared the accuracy of diagnosis of three methods of biopsy for extremities soft tissue tumors and concluded that open incisional biopsy was $100 \%$ accurate in establishing the exact diagnosis, while FNA had a $33.3 \%$ accuracy and CNB had $45.6 \%$ accuracy (5). Incisional biopsy has been the gold standard for soft tissue tumors diagnosis, with an accuracy of $94 \%$ to $100 \%$, however, it is expensive and carries a complication rate of up to $16 \%$, including hematoma and wound problems that may interfere with future treatments (14). As the soft tissue sarcoma enlarges, it outgrows of its blood supply, leading to area of central necrosis. Closed biopsy may result in obtaining the necrotic material (5).

\section{Conclusions}

Biopsy is a final diagnostic procedure and should be delayed until completion of clinical evaluation and imaging studies. Biopsy is indicated in malignant and uncertain lesions. FNA has the highest accuracy in homogenous tumor. It can also be used for cytological confirmation of local or distant recurrence. CNB is usually selected as the first biopsy modality. Because of heterogeneity of bone and soft tissue tumor, multiple cores should be obtained in different directions. Incisional biopsy is indicated in difficult cases when a larger specimen is necessary for diagnosis. In malignant bone tumors, the soft tissue component is as much representative as the bony component. The specimen should be obtained by knife or large head curette to avoid crushing the tissue's texture. Disrupting the cortex increases the risk of pathologic fracture.

\section{References}

1. Bickels J, Jelinek JS, Shmookler BM, Neff RS, Malawer MM. Biopsy of musculoskeletal tumors. Current concepts. Clin Orthop Relat Res.1999(368):212-9.

2. Dorfman HD, Czerniak B. General Considerations. Dorfman HD, Czerniak B editors. St Louis: CV Mosby; 1998.

3. Mankin HJ, Mankin CJ, Simon MA. The hazards of the biopsy, revisited. Members of the Musculoskeletal Tumor Society. J Bone Joint Surg Am. 1996;78(5):656-63.

4. Tyagi R, Dey P. Needle tract seeding: an avoidable complication. Diagn Cytopathol. 2014;42(7):636-40.

5. Kasraeian S, Allison DC, Ahlmann ER, Fedenko AN, Menendez LR A comparison of fine-needle aspiration, core biopsy, and surgical biopsy in the diagnosis of extremity soft tissue masses. Clin Orthop Relat Res. 2010;468(11):2992-3002.

6. Enneking WF. General Principles of Musculoskeletal Tumor Surgery. Enneking WF editor. New York: Churchill- Livingstone; 1983.

7. Yang YJ, Damron TA. Comparison of needle core biopsy and fineneedle aspiration for diagnostic accuracy in musculoskeletal lesions. Arch Pathol Lab Med. 2004;128(7):759-64.

8. Ayala AG, Ro JY, Fanning CV, Flores JP, Yasko AW. Core needle biopsy and fine-needle aspiration in the diagnosis of bone and soft-tissue lesions. Hematol Oncol Clin North Am. 1995;9(3):633-51.

9. Mehrotra R, Singh M, Singh PA, Mannan R, Ojha VK, Singh P Should fine needle aspiration biopsy be the first pathological investigation in the diagnosis of a bone lesion? An algorithmic approach with review of literature. Cytojournal. 2007;4:9.

10. Pohlig F, Kirchhoff C, Lenze U, Schauwecker J, Burgkart R, Rech $\mathrm{H}$, von Eisenhart-Rothe R, et al. Percutaneous core needle biopsy versus open biopsy in diagnostics of bone and soft tissue sarcoma: a retrospective study. Eur J Med Res. 2012;17:29.

11. Skrzynski MC, Biermann JS, Montag A, Simon MA. Diagnostic accuracy and charge-savings of outpatient core needle biopsy compared with open biopsy of musculoskeletal tumors.J Bone Joint Surg Am. 1996;78(5):644-9.

12. Clark CR, Morgan C, Sonstegard DA, Matthews LS. The effect of biopsy-hole shape and size on bone strength.J Bone Joint Surg Am. 1977;59(2):213-7.

13. Rydholm A, Berg NO, Gullberg B, Thorngren KG, Persson BM. Epidemiology of soft-tissue sarcoma in the locomotor system. Acta Pathol Microbiol Immunol Scand. 1984;92:363-74.

14. Shiu MH, Castro EB, Hajdu SI, Fortner JG. Surgical treatment of 297 soft tissue sarcomas of the lower extremity. Ann Surg. 1975;182(5):597-602. 